“( 2013 IEEE. Personal use of this material is permitted. Permission from IEEE must be obtained for all other uses, in any current or future media, including

reprinting/republishing this material for advertising or promotional purposes, creating new collective works, for resale or redistribution to servers or lists, or reuse of any copyrighted component of this work in other works." 


\title{
Breast Cancer Detection in Highly Dense Numerical Breast Phantoms Using Time Reversal
}

\author{
Md. Delwar Hossain ${ }^{1} \quad$ Ananda Sanagavarapu Mohan ${ }^{2}$
}

\begin{abstract}
In this paper we investigate the detection of breast cancer using two-dimensional slices of realistic numerical phantoms employing time reversal microwave imaging. We used maximum-likelihood estimation coupled with time reversal technique to detect and estimate the location of tumor using FDTD based breast phantoms that contain dense fibroglandular tissue clutter. We show that time reversal maximum-likelihood estimation can detect and accurately localize tumors even in highly dense breasts where the dielectric contrast between healthy dense breast tissue and cancerous lesions is quite low without requiring any contrast enhancing agents.
\end{abstract}

\section{INTRODUCTION}

Breast cancer is one of the dominant causes of morbidity and mortality among women. It has been demonstrated that early detection is the single most significant predictor of long term survival. Therefore, improvements in detection can greatly help to increase the cure rate of breast cancer. Currently, Xray mammography is the gold standard tomography for breast cancer imaging and has significantly reduced the mortality rate. However, mammography has several drawbacks such as high false-positive and false-negative rates, ionizing radiation, poor accuracy in the presence of glandular tissue, and causes pain and discomfort due to applied compression. Two other clinically available techniques viz., magnetic resonance imaging (MRI) and ultrasound imaging have limitations either in terms of cost, or achievable resolution and ability to separate benign and malignant tissues. Breast is composed of fatty adipose and dense glandular tissues. With age dense glandular tissues diminish. Hence it is quite challenging to detect breast tumors in younger women owing to the presence of dense glandular tissues. Although breast cancer risk increases with age, it is not uncommon for younger women to develop breast cancer.

The time reversal (TR) method [1] for breast cancer detection has been well established [2-7]. Radar based TR microwave imaging technique is sensitive to the dielectric contrast between healthy and malignant breast tissues [8]. When the dielectric contrast between healthy and malignant tissues is low, TR methods often tend to fail to detect the presence of tumor. Recently contrast aiding agents have been suggested to raise the dielectric contrast to achieve detection and classification between malignant and benign tissues for microwave imaging techniques $[9,10]$.
In this paper we employ efficient beamforming techniques coupled with TR imaging for improving the localization of malignant lesions in dense breast phantoms. We employ maximum-likelihood estimation with TR for the detection of tumor inside realistic dense numerical breast phantom and compare its performance with conventional method known as decomposition of the time reversal operator (DORT) and TR MUSIC techniques. Maximumlikelihood based TR imaging was first proposed by Shi and Nehorai [1] and was later employed for detection in simplified breast models in [7]. But the deterministic breast model in [7] uses simplified approximations for breast tissue heterogeneities and also neglected the effects of multiple scattering among different types of tissues. In this study we use realistic numerical breast phantoms representing true breast tissue heterogeneity[8]. Our results indicate that time reversal maximum-likelihood (TR ML) has superior performance over DORT and TR MUSIC techniques.

The rest of the paper is organized as follows. In section 2 we describe TR ML imaging technique along with conventional DORT and TR MUSIC imaging techniques. A brief description of the FDTD simulation setup as well as the breast mimicking numerical phantom is presented in section 3 . We present the results on breast cancer detection in section 4 and the conclusions are provided in section 5 .

\section{TIME REVERSAL MICROWAVE IMAGING TECHNIQUES}

Synthetic TR microwave imaging techniques use the multistatic data matrix (MDM) which is formed by transmitting short pulse from each element of the transmitting array and the resulting scattered field is recorded by the receiving array. Let us consider that an $N$ element UWB transceiver array is employed to localize $M$ targets in a 2-D space. When the $j$-th element of the array is excited using the electric field $e_{j}(\omega)$, the incident field is scattered by the $M$-targets. The scattered field is recorded by the $l$-th element of the array can be expressed as

$$
E_{l j}^{s}(\omega)=\sum_{m=1}^{M} G\left(\mathbf{x}_{m}, \mathbf{r}_{l}, \omega\right) \tau_{m}(\omega) G\left(\mathbf{r}_{j}, \mathbf{x}_{m}, \omega\right) e_{j}(\omega)(1)
$$

where $\tau_{m}(\omega)$ is a tensor that represents the backscattering strength as a function of angular frequency, $\mathbf{x}_{m}$ is the location of $m$-th target, $\mathbf{r}_{j}$ is the $j$ -

\footnotetext{
${ }^{1}$ Centre for Health Technologies, Faculty of Engineering and IT, University of Technology Sydney, Australia e-mail: Md.D.Hossain@student.uts.edu.au.

${ }^{2}$ Centre for Health Technologies, Faculty of Engineering and IT, University of Technology Sydney, Australia e-mail: Ananda.Sanagavarapu@uts.edu.au.
} 
th receiving element location, $k$ is the wave number and $G$ is Green's function of the background medium. Hence, the $l, j$-th element of the MDM can be written as

$$
\kappa_{l j}=\left\{E_{l, j}^{s}\right\}=\sum_{m=1}^{M} \tau_{m} \mathbf{g}_{r}\left(\mathbf{x}_{m}, \omega\right) \mathbf{g}_{t}^{T}\left(\mathbf{x}_{m}, \omega\right)
$$

where, ( $)^{T}$ denotes transpose operation and Green's function vectors $\mathbf{g}_{r}$ and $\mathbf{g}_{t}$ are defined as

$$
\begin{aligned}
& \mathbf{g}_{r}\left(\mathbf{x}_{m}, \omega\right)=\left[G\left(\mathbf{r}_{1}, \mathbf{x}_{m}, \omega\right), \quad \ldots \quad, G\left(\mathbf{r}_{N}, \mathbf{x}_{m}, \omega\right)\right](3) \\
& \mathbf{g}_{t}\left(\mathbf{x}_{m}, \omega\right)=\left[G\left(\mathbf{x}_{m}, \mathbf{r}_{1}, \omega\right), \cdots, G\left(\mathbf{x}_{m}, \mathbf{r}_{N}, \omega\right)\right](4)
\end{aligned}
$$

where, subscripts $t$ and $r$ are used for transmission and reception respectively. For a transceiver array and for a reciprocal medium, the MDM becomes symmetric

$$
\kappa_{l j}(\omega)=\kappa_{j l}(\omega)
$$

In the presence of white Gaussian noise we can represent the measured multistatic matrix as

$$
\mathbf{K}=\mathbf{K}+\sigma^{2} \mathbf{I}
$$

where, $\mathbf{I}$ is an $N \times N$ identity matrix and $\sigma^{2}$ is the noise variance. Using singular value decomposition $\mathbf{K}$ can be represented as

$$
\mathbf{K}(\omega)=\mathbf{U}(\omega) \boldsymbol{\Phi}(\omega) \mathbf{V}^{H}(\omega)
$$

The TR operator is defined as

$$
\mathbf{T}=\mathbf{K}^{H} \mathbf{K}
$$

where ()$^{H}$ denotes conjugate transpose operation. Substituting (7) into (8) we get

$$
\mathbf{T}=\mathbf{V}(\omega) \boldsymbol{\Phi}(\omega) \mathbf{U}^{H}(\omega) \mathbf{U}(\omega) \boldsymbol{\Phi}(\omega) \mathbf{V}^{H}(\omega)
$$

Since $\mathbf{U}$ and $\mathbf{V}$ are both unitary matrix

$$
\mathbf{T}=\mathbf{V}(\omega) \boldsymbol{\Phi}^{2}(\omega) \mathbf{V}^{H}(\omega)
$$

Hence, we can use singular value decomposition of $\mathbf{K}$ instead of using eigen value decomposition of $\mathbf{T}$.

\subsection{DORT Imaging}

Each significant eigen value of $\Phi_{m}^{2}$ represents the presence of a target in the region under investigation. These targets can be identified by back propagation of their corresponding eigen vectors. The eigen vectors are related to the Green's function vector of the medium as [11]

$$
\mathbf{v}_{m}(\omega) \approx e^{j \varphi(\omega)} \frac{\mathbf{g}^{*}\left(\mathbf{x}_{m}\right)}{\left\|\mathbf{g}\left(\mathbf{x}_{m}\right)\right\|}
$$

where, $\varphi(\omega)$ is the phase as a function of angular frequency arising from singular value decomposition and ()$^{*}$ denotes conjugate operation.

Consequently the DORT imaging function that utilizes the signal subspace is formed as

$$
I_{D}(\mathbf{r})=\sum_{n=1}^{M}\left|\left\langle\mathbf{v}_{n}^{*} \mid \mathbf{g}(\mathbf{r}, \omega)\right\rangle\right|^{2}
$$

\subsection{Time Reversal MUSIC Imaging}

Ideally, the signal subspace and noise subspace are orthogonal to each other. As a result at the target location the inner product of the Green's function vector with the eigen vectors that span the noise subspace is ideally zero. Hence, TR MUSIC can produce high resolution images using the following imaging function

$$
I_{T R M}(\mathbf{r})=\frac{1}{\sum_{n=M+1}^{N}\left|\left\langle\mathbf{v}_{n}{ }^{*} \mid \mathbf{g}(\mathbf{r}, \omega)\right\rangle\right|^{2}}
$$

\subsection{Time Reversal Maximum Likelihood Imaging}

TR imaging is known to have near-far problem if the target is located far away from the transceiver array [1]. Maximum likelihood estimation overcomes this problem and as a result superior imaging performance is obtained even when the target is far away or even surrounded by highly dense clutter. The maximum likelihood estimation of the target location and scattering strength can be obtained from the minimization problem

$$
\hat{\mathbf{x}}_{m}, \hat{\tau}_{m}=\underset{\hat{\mathbf{x}}_{m}, \hat{\tau}_{m}}{\arg \min }\left\|\mathbf{K}-\mathbf{K}\left(\mathbf{x}_{m}, \tau_{m}\right)\right\|_{\mathrm{F}}^{2}
$$

(8) Subsequently we obtain the following imaging function for TR ML estimation [1]

$$
I_{T R M L}(\mathbf{r})=\frac{1}{\operatorname{Tr}\left(\mathbf{P}_{\mathbf{g}(\mathbf{r})}^{\perp} \operatorname{vec}(\mathbf{K})\right)}
$$

\section{FDTD SIMULATION USING NUMERICAL BREAST PHANTOM}

We carried out FDTD simulation on 2-D slices of realistic breast models obtained from the UWCEM numerical breast phantom repository [12]. A 2-D slice (TM-z) from a highly dense breast phantom is used in FDTD simulation as shown in Figure 1. A 21element transceiver array of infinite line sources is employed. The location of array elements is indicated using the blue dots. The 2-D plane is indicated in blue colour as shown in Figure 1. The excitation is a differentiated Gaussian pulse with $-3 \mathrm{~dB}$ bandwidth 1$5 \mathrm{GHz}$ and $\mathrm{TM}-z$ polarized. We use uniform grid size of $0.5 \mathrm{~mm}$ in all directions. The breast tissue dielectric properties are obtained from [8]. In order to minimize the reflections from skin the breast phantom along with the transceiver array is immersed in a matching liquid. Skin artifact has also been removed using entropy based algorithm [13]. 


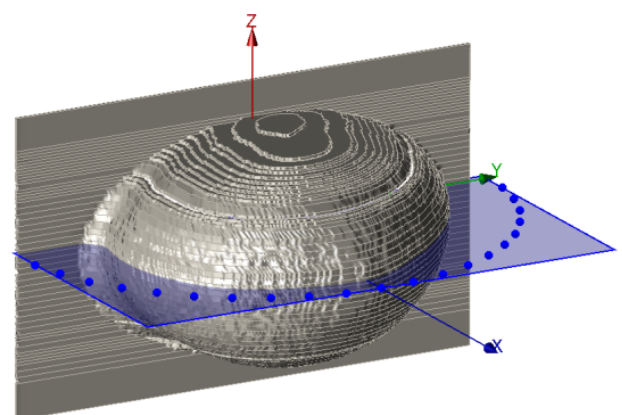

Figure 1: Numerical breast phantom for FDTD simulation.

\section{TIME REVERSAL IMAGING RESULTS}

We present the imaging results for different TR techniques employing highly dense and heterogeneously (moderately) dense breast phantoms. Figures 2-4 show the results in heterogeneously dense breast phantom while Figures 5-7 show results in highly dense breast phantom. All these images were obtained at $\mathrm{SNR}=20 \mathrm{~dB}$. In all the images the assumed tumor location is indicated with a small white circle.

It is observed that for heterogeneously dense breast phantom DORT image quite accurately estimates the tumor location although its resolution is low. TR MUSIC imaging produces strong intensity near the true tumor location. However, there are several minor peaks scattered throughout the image. This leads to confusion regarding estimation of the tumor location and may result in false positives. TR ML result is quite impressive for heterogeneously dense breast phantom as shown in Figure 4. For TR ML technique the tumor location is very much accurately represented which is less affected by the presence of dense glandular tissues in the breast compared with DORT and TR MUSIC images.

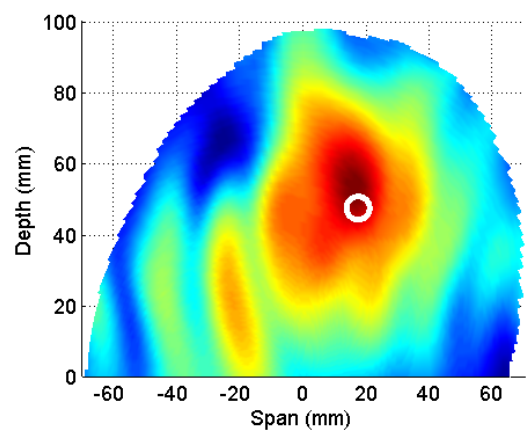

Figure 2: DORT image for heterogeneously dense phantom.

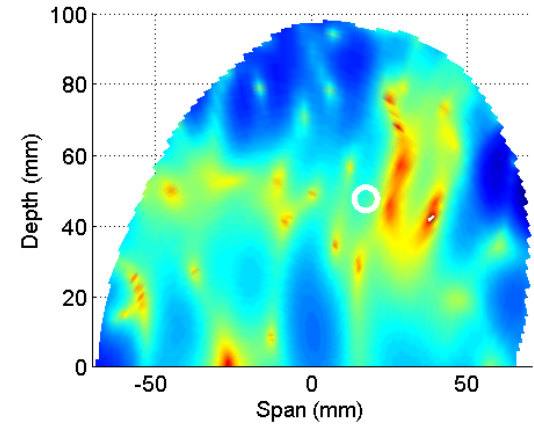

Figure 3: TR MUSIC image for heterogeneously dense phantom.

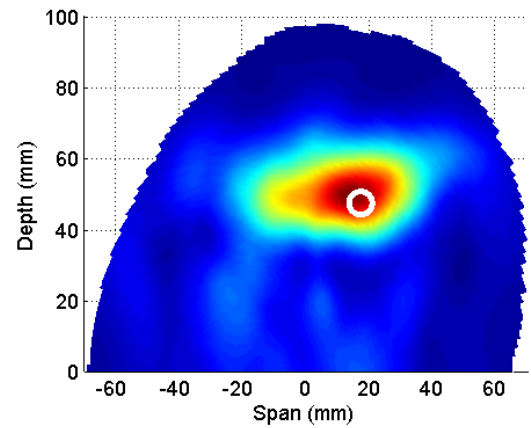

Figure 4: TR ML image for heterogeneously dense phantom.

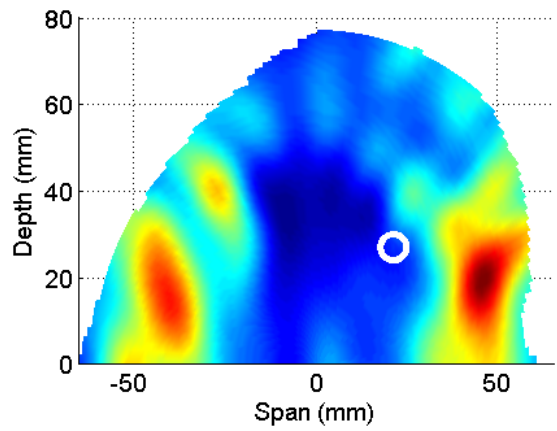

Figure 5: DORT image for highly dense phantom.

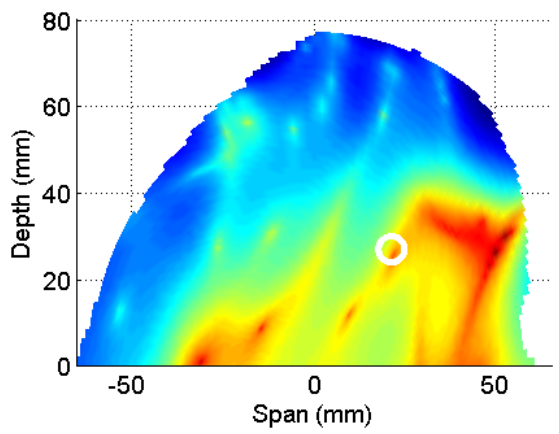

Figure 6: TR MUSIC image for highly dense phantom. 


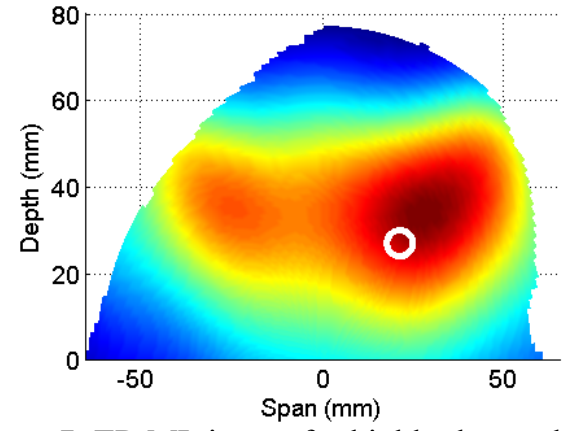

Figure 7: TR ML image for highly dense phantom.

We observe similar trends in imaging results for highly dense breast phantoms. The highly dense breast phantom is almost entirely composed of dense tissues, and so both DORT and TR MUSIC fail to accurately localize the tumor. TR ML imaging on the other hand still successfully estimates the tumor location but the imaging resolution becomes poorer as the scattering from dense glandular tissues interfere with the scattering from the tumor.

\section{CONCLUSIONS}

It is observed that TR ML imaging can localize tumor even in the highly dense breasts where DORT and TR MUSIC techniques fail. The superiority of TR ML is readily realized from highly dense breast phantom results. As TR ML estimation overcomes the near far problem of conventional TR imaging techniques, tumor even in very highly dense breast phantom can be localized.

\section{Acknowledgments}

The work reported in this paper is supported by the Australian Research Council through a Discovery Grant DP 0773234.

\section{References}

[1] S. Gang and A. Nehorai, "A Relationship Between Time-Reversal Imaging and MaximumLikelihood Scattering Estimation," IEEE Trans. Signal Process., vol. 55, pp. 4707-4711, 2007.

[2] P. Kosmas, "Application of the DORT technique to FDTD-based time reversal for microwave breast cancer detection," in Proc. European Microw. Conf., 2007, pp. 306-308.

[3] P. Kosmas and C. M. Rappaport, "FDTD-based time reversal for microwave breast cancer detection-localization in three dimensions," IEEE Trans. Microw. Theory Tech., vol. 54, pp. 19211927, 2006.

[4] Y. Chen, E. Gunawan, K. S. Low, S. C. Wang, C. B. Soh, and T. C. Putti, "Time-reversal ultrawideband breast Imaging: pulse design criteria considering multiple tumors with unknown tissue properties," IEEE Trans. Antennas Propag., vol. 56, pp. 3073-3077, 2008.

[5] M. H. S. Sajjadieh and A. Asif, "Unsupervised Time Reversal Based Microwave Imaging for Breast Cancer Detection," in 24th Canadian Conf. Elect. Comput. Eng. (CCECE), 2011, pp. 001411001415.

[6] J. Yuanwei, J. M. F. Moura, J. Yi, M. Wahl, Z. He, and H. Qiuhong, "Breast cancer detection by time reversal imaging," in Proc. 5th IEEE Int. Symp. Biomed. Imag.: From Nano to Macro, 2008, pp. 816-819.

[7] M. J. Abedin and A. S. Mohan, "ML based time reversal microwave imaging for the localisation of breast tissue malignancies," in Proc. IEEE Antennas Propag. Society Int. Symp. (APS/URSI), 2010, pp. 1-4.

[8] M. Lazebnik, et al., "A large-scale study of the ultrawideband microwave dielectric properties of normal, benign and malignant breast tissues obtained from cancer surgeries " Phys. Med. Biol., vol. 52, pp. 6093-6115, 2007.

[9] A. Mashal, B. Sitharaman, X. Li, P. K. Avti, A. V. Sahakian, J. H. Booske, and S. C. Hagness, "Toward Carbon-Nanotube-Based Theranostic Agents for Microwave Detection and Treatment of Breast Cancer: Enhanced Dielectric and Heating Response of Tissue-Mimicking Materials," IEEE Trans. Biomed. Eng., vol. 57, pp. 1831-1834, 2010.

[10] Y. Chen and P. Kosmas, "Detection and Localization of Tissue Malignancy Using Contrast-Enhanced Microwave Imaging: Exploring Information Theoretic Criteria," IEEE Trans. Biomed. Eng., vol. 59, pp. 766-776, 2012.

[11]A. J. Devaney, "Time reversal imaging of obscured targets from multistatic data," IEEE Trans. Antennas Propag., vol. 53, pp. 1600-1610, 2005.

[12] UWCEM breast phantom repository, available: http://uwcem.ece.wisc.edu/MRIdatabase/index.ht $\mathrm{ml}$

[13]Z. Wanjun and F. Chin, "Entropy-based time window for artifact removal in UWB imaging of breast cancer detection," IEEE Signal Process. Lett., vol. 13, pp. 585-588, 2006. 